\title{
New Approaches to Molecular Imaging of Multiple Myeloma
}

\author{
Ravi Vij ${ }^{1}$, Kathryn J. Fowler ${ }^{2}$, and Monica Shokeen ${ }^{2}$ \\ ${ }^{1}$ Division of Hematology and Oncology, Washington University School of Medicine, Saint Louis, Missouri; and ${ }^{2}$ Mallinckrodt Institute \\ of Radiology, Washington University School of Medicine, Saint Louis, Missouri
}

\begin{abstract}
Molecular imaging plays an important role in detection and staging of hematologic malignancies. Multiple myeloma (MM) is an age-related hematologic malignancy of clonal bone marrow plasma cells characterized by destructive bone lesions and is fatal in most patients. Traditional skeletal survey and bone scans have sensitivity limitations for osteolytic lesions manifested in MM. Progressive biomedical imaging technologies such as low-dose CT, molecularly targeted PET, MRI, and the functionalanatomic hybrid versions (PET/CT and PET/MRI) provide incremental advancements in imaging MM. Imaging with PET and MRI using molecularly targeted probes is a promising precision medicine platform that might successfully address the clinical ambiguities of myeloma spectrum diseases. The intent of this focus article is to provide a concise review of the present status and promising developments on the horizon, such as the new molecular imaging biomarkers under investigation that can either complement or potentially supersede existing standards.
\end{abstract}

Key Words: multiple myeloma (MM); monoclonal gammopathy of undetermined significance (MGUS); PET/CT; PET/MRI; receptor very late antigen-4 (VLA-4); metabolic imaging

J Nucl Med 2016; 57:1-4

DOI: $10.2967 /$ jnumed.115.163808

Multiple myeloma (MM), the second most common age-related hematologic malignancy in the United States, is incurable in most patients. MM is a malignancy of clonal bone marrow plasma cells whose DNA has undergone the characteristic class-switch recombination and somatic hypermutation (1). In addition to hallmark genetic mutations, bone microenvironmental elements play a critical role in the pathogenesis of MM (2). MM is preceded by a premalignant stage called monoclonal gammopathy of undetermined significance (MGUS), with an incidence of progression to MM of $0.5 \%-1 \%$ per year (3). Smoldering MM is an intermediate clinical stage in which the risk of progression to $\mathrm{MM}$ is $10 \%$ per year (3). The diagnostic criteria of the International Myeloma Working Group for premalignant and malignant MM have been elegantly summarized by Rajkumar et al. (3). Because the U.S. population is aging, there is expected to be an increase in the incidence of MM, along with the associated costs. Total health care costs in the first year after diagnosis of MM are $\$ 118,353$ (4). Advancements in targeted therapy as well as the success of stem cell transplantation have contributed to improvements in the 5-y survival rate in MM (26.3\% in 1975 vs. $46.6 \%$ in 2011) (5). Promising new agents are currently under development for relapsed and refractory

\footnotetext{
Received Jul. 14, 2015; revision accepted Nov. 2, 2015.

For correspondence or reprints contact: Monica Shokeen, Mallinckrodt Institute of Radiology, Department of Radiology, 4525 Scott Ave., St. Louis, MO 63110.

E-mail: mshokeen@wustl.edu

Published online Nov. 5, 2015

COPYRIGHT (c) 2016 by the Society of Nuclear Medicine and Molecular Imaging, Inc.
}

MM (6). The treatment regimen for MM is dictated by patient eligibility for autologous or allogeneic stem cell transplantation. About $80 \%$ of MM patients treated at our institution are transplant-eligible. Most of these patients receive combination therapy with bortezomib (a proteasome inhibitor), lenalidomide (an immunomodulatory drug), and dexamethasone (a corticosteroid), although treatment is tailored around patient age and comorbidities. Despite the improvement in 5-y survival, relapse and acquired drug resistance remain a challenge in MM. Remissions are transient, and most patients eventually experience a relapse and die from progressive disease. The mechanisms by which premalignant myeloma (MGUS and smoldering MM) progresses to MM are complex and not fully known. Malignant myeloma plasma cells accumulate in the bone marrow and disrupt bone homeostasis, leading to bone destruction and marrow failure (Fig. 1). Consequently, the risk of related skeletal events such as fractures is high in MM patients and continues to rise even with treatment (7). Malignant plasma cells are generally avid secretors of immunoglobulins; therefore, MM and its obligate precursor state, MGUS, are readily detected in most cases using serum markers or urine markers (either intact immunoglobulin or free light chains). However, serum markers are insufficient to distinguish premalignant MGUS and smoldering MM from fully transformed MM. The diagnosis of MM requires a high monoclonal tumor burden or end organ damage such as lytic bone lesions. Evaluation of progression and treatment response is also confounded in the $10 \%$ of MM patients who display an oligosecretory phenotype (defined as serum M-protein $<1 \mathrm{~g} / \mathrm{dL}$ and urine M-protein $<200 \mathrm{mg} / 24 \mathrm{~h})(8)$. The timely and accurate diagnosis of MM is important; a delay can be detrimental to the patient's outcome. Imaging might provide critical information such as predicting high-risk fracture sites, visualizing nonsecretory and oligosecretory MM tumors, and assessing treatment response at various stages of disease (9).

The current clinical practice for MM includes an initial diagnostic full-skeleton radiographic survey for lytic bone lesions (recommended by the International Staging System) (10). This survey involves acquiring a series of radiographs (plain 2-dimensional films) to cover the entire skeleton or common anatomic regions appropriate for clinical indications of the whole spine. Despite the advantage of this fast, relatively low-cost imaging option, a key limitation of the radiographic skeletal survey is its low sensitivity to early osteolytic lesions, as lesions typically can be detected only after 30\%-50\% of mineralized bone destruction has occurred (11). Low-dose whole-body (WB) CT is now frequently used in MM and has higher sensitivity than radiographs for superimposed skeletal regions such as the scapulae, ribs, and sternum (12). Additionally, $\mathrm{CT}$ is better than conventional radiography for detecting extraosseous lesions and for radiotherapy planning (13). PET and MRI have high sensitivity and specificity for providing molecular, functional, and metabolic information on MM patients. Recent advances in functional PET and MRI for MM are discussed below. 


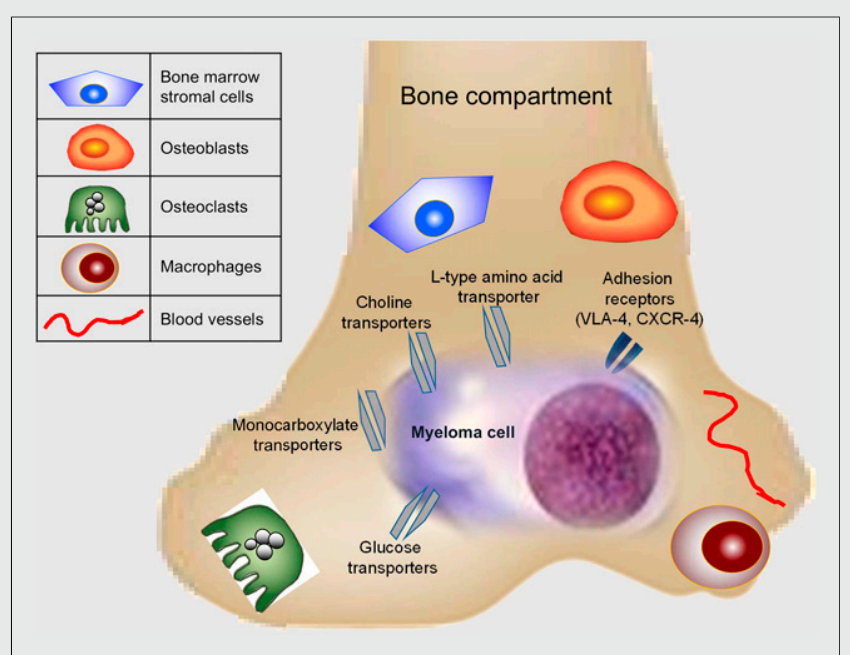

FIGURE 1. Simplified overview of molecular markers targeted by PET and MRI. MM cells and microenvironment possess anatomic and functional biomarkers for imaging. Myeloma cells primarily reside in bone marrow compartment, disrupting bone microenvironment and altering metabolism. CXCR-4 = chemokine receptor 4 .

\section{PET IMAGING IN MM}

Functional PET imaging is widely used to assess medullary and extramedullary disease, providing diagnostic factors such as standardized uptake value $\left(\mathrm{SUV}_{\max }\right.$ or $\left.\mathrm{SUV}_{\text {mean }}\right)$, quantifying the number of focal lesions, and identifying diffuse bone marrow infiltration. Metabolism in cancer cells is altered as compared with normal cells. PET imaging of tumor metabolism using ${ }^{18} \mathrm{~F}$-FDG has been widely applied in the clinic for staging disease, planning treatment, and monitoring response (14). Several reviews on the imaging of tumor cell metabolism are available, including a comprehensive article by Plathow and Weber (15). Although most clinical metabolic PET imaging in MM is performed with ${ }^{18} \mathrm{~F}$-FDG, ${ }^{18} \mathrm{~F}-\mathrm{FDG}$ has significant limitations for MM. MM cells are hypoproliferative, do not consistently overexpress glucose transporter 1 , and ${ }^{18} \mathrm{~F}-\mathrm{FDG}$ does not easily distinguish between a benign lesion and a low-metabolism MM lesion. Over a third of intramedullary myeloma lesions can go undetected by ${ }^{18} \mathrm{~F}$-FDG PET (16). There is an unmet need for myeloma-specific diagnostic imaging agents. New tracers targeting different molecular signatures, and therefore biologic properties of myeloma, will enhance knowledge of disease progression and lead to personalized patient management.

\section{${ }^{11} \mathrm{C}$-Acetate PET}

A variety of cancer cells, including myeloma cells, can metabolize exogenous acetate for de novo membrane biosynthesis through fatty acid synthase and enter the tricarboxylic acid cycle (17). Fatty acid synthase is overexpressed in MM cells and has been shown to sustain the biogenesis of lipids from extracellular acetate (18). Okawa et al. have shown the expression of fatty acid synthase in primary myeloma cells as well as in cell lines and demonstrated apoptosis upon pharmacologic inhibition of fatty acid synthase in vitro (19). ${ }^{11} \mathrm{C}$-acetate is a promising clinical PET tracer that has been shown to be sensitive in bone metastases, primarily prostate cancer, and is being evaluated for cancers that have limited avidity for ${ }^{18} \mathrm{~F}$-FDG (20). Clinically, in a small prospective study Lin et al. showed a significant correlation between systemic tumor burden as measured by percentage of bone marrow plasma cell infiltrates and ${ }^{11} \mathrm{C}$-acetate marrow uptake $(r=$ $0.63 ; P=0.01$ ), and a higher number of focal lesions were detected with ${ }^{11} \mathrm{C}$-acetate than with ${ }^{18}$ F-FDG (13 vs. 10$)(21)$. Ho et al. dem- onstrated that ${ }^{11} \mathrm{C}$-acetate had enhanced sensitivity over ${ }^{18} \mathrm{~F}-\mathrm{FDG}$ (84.6\% vs. $57.7 \%)$ in detecting diffuse infiltration and focal lesions in MM patients. The same group also demonstrated a correlation between ${ }^{11} \mathrm{C}$-acetate marrow uptake and clinical serum $\beta_{2}$-microglobulin levels, as well as a posttreatment reduction in ${ }^{11} \mathrm{C}$-acetate uptake that was associated with systemic measures of response (22). These data support additional ${ }^{11} \mathrm{C}$-acetate PET and ${ }^{18} \mathrm{~F}$-FDG PET comparison studies in patients with newly diagnosed or refractory disease.

\section{${ }^{11} \mathrm{C} /{ }^{18} \mathrm{~F}$-Choline PET}

Radiolabeled choline $\left({ }^{11} \mathrm{C}\right.$ or $\left.{ }^{18} \mathrm{~F}\right)$ and its analogs are precursors for biosynthesis of cellular membrane phospholipids and are used as metabolic PET markers of membrane metabolism and turnover. In a small study of 10 patients, Nanni et al. reported ${ }^{11} \mathrm{C}$-choline to be better than ${ }^{18} \mathrm{~F}$-FDG at identifying myeloma lesions in the bone (37 vs. 22) (23). There have been reports of incidental findings of MM or a solitary plasmacytoma by radiolabeled choline PET (24). Additional preclinical and clinical evaluations will help correlate myeloma hallmarks with choline metabolism and uptake mechanisms.

\section{Amino Acid PET}

Probes targeted to amino acid transporters represent a promising class of imaging agents in view of their ability to reveal increased rates of amino acid transport by cancer cells (25). Tumor uptake of amino acid tracers primarily reflects the rate and mechanism of transport rather than other metabolic fates such as protein synthesis. ${ }^{11} \mathrm{C}$-methionine is a potential amino acid PET tracer for MM (26). Luckerath et al. demonstrated in myeloma cells a significantly higher uptake of radiolabeled methionine than of ${ }^{18} \mathrm{~F}-\mathrm{FDG}$, and there was differential methionine uptake in myeloma cell lines (with high uptake in cell lines of worse prognosis) (27). L-type amino-acid transporter 1 (LAT-1) mediates sodium-independent cellular transport of amino acids for protein synthesis and other metabolic pathways, and high levels of LAT-1 correlate with proliferating cancers. Isoda et al. have demonstrated expression of LAT-1 by immunohistochemistry in $100 \mathrm{MM}$ patients and found LAT-1 in $56 \%$ of patients (28). The ${ }^{18}$ F-labeled amino acid 3,4-dihydroxy-6- ${ }^{18}$ F-fluoro-L-phenylananine is a tracer for imaging LAT-1 and warrants evaluation as a PET marker of prognosis and therapeutic planning and response in MM.

\section{Receptor-Targeted PET}

MM resculpts the bone microenvironment by facilitating neoangiogenesis, recruitment of tumor-associated macrophages, and activation of osteoclasts while inhibiting osteoblasts, thereby causing a vicious cycle of tumor growth and bone destruction. A grim result of this interplay is that most MM patients are diagnosed only after pathologic bone fracture has occurred. Integrins are glycoprotein cell receptors that transmit signals bidirectionally across the plasma membrane by undergoing conformational changes in response to stimuli from intracellular products and extracellular components (29). Interactions between integrins on the surface of tumor cells and the stromal environment play a defining role in the pathogenesis of MM. The activated form of the receptor VLA-4 (very late antigen 4, also known as integrin $\alpha_{4} \beta_{1}$ ) is present at high levels on MM cells. VLA-4 is a critical mediator of myeloma cell adhesion to the bone marrow stroma and promotes MM cell trafficking, proliferation, and drug resistance. We previously demonstrated sensitive and specific molecular imaging of activated VLA-4 in MM tumors using the PET radiopharmaceutical ${ }^{64} \mathrm{Cu}-\mathrm{CB}-\mathrm{TE} 1 \mathrm{~A} 1 \mathrm{P}-\mathrm{LLP} 2 \mathrm{~A}(30)$. We currently are developing VLA-4-targeted radiopharmaceuticals for translation into humans to image myeloma spectrum diseases and compare with ${ }^{18}$ F-FDG PET. Chemokine receptor 4 is another key receptor that 
plays an important role in MM pathogenesis. Philipp-Abbrederis et al. recently demonstrated imaging of advanced MM in humans using the chemokine receptor 4-targeted PET probe ${ }^{68} \mathrm{Ga}$-pentixafor (31).

\section{MRI IN MM}

The role of MRI in imaging MM relies on 2 primary functions: improved sensitivity for detecting pathologic lesions, and the potential for predictive and prognostic imaging biomarkers. With regard to sensitivity of disease detection, WB MRI offers high soft-tissue contrast and high spatial resolution, which in turn yield sensitivity superior to that of conventional radiography for visualization of focal and diffuse tumor infiltration of bone marrow in untreated patients (32). The updated criteria for diagnosis of MM by the International Myeloma Working Group recommend MRI as part of the initial assessment (3), and MRI is also considered particularly beneficial in patients with smoldering MM (33). Hillengass et al., in a study of 149 patients with asymptomatic MM, demonstrated that patients with more than one focal lesion had a significantly shorter progression-free survival than those without a focal lesion or with only one $(P<0.001)$ (34). Beyond sensitivity, there has been much interest in developing prognostic and predictive imaging biomarkers using the functional capabilities of MRI. One such example is dynamic contrast-enhanced (DCE) MRI using gadolinium-based contrast agents. Increased angiogenesis of the bone marrow is associated with the transition from premalignant states to MM. In a prospective clinical trial, 30 patients were evaluated for level of angiogenesis from MGUS to frank malignancy (35). The kinetic parameters $K^{\text {trans }}$ (transendothelial transport of gadolinium from the vascular compartment to the tumor interstitium [wash in]) and $K_{\text {ep }}$ (reverse transport of gadolinium back into the vascular space [washout]) derived from DCE MRI of the lumbar vertebrae were compared with bone marrow microvessel density and a serum panel of 17 angiogenic markers. The study found a moderateto-strong correlation between marrow microvessel density and $K_{\text {ep }}$ in all patients $(r=0.59 ; P=0.001)$ and a weak-to-moderate correlation between marrow microvessel density and $K^{\text {trans }}$ in all patients $(r=$ $0.43 ; P=0.03$ ). It should be noted that DCE is not a WB application and is done to evaluate a specific anatomic region such as in the case of a plasmacytoma. To evaluate the cellularity of a lesion or to quantify the distribution of plasma cells in bone marrow, apparent diffusion coefficients (ADCs) derived from diffusion-weighted imaging (DWI) sequences are used. DWI can noninvasively quantify altered diffusion, volume, and flow permeability in new vessels. The relationship between tumor and background ADCs in marrow is complex and depends on the degree of marrow activation and the status of the tumor. In a pilot study of 11 patients with metastatic osseous lesions, median global ADCs acquired by semiautomated segmentation of DWI data allowed for differentiation of responders from nonresponders (36). A prospective trial of 26 patients with MM and baseline/follow-up WB DWI found a significant change in posttherapy ADCs that was reproducible between multiple interpreters (37). A few additional studies have shown similar results suggesting that ADC DWI is a potential response biomarker platform (38-40). Although ADC DWI data provide insight into tumor cellularity and disease activity, the interpretation of these images can be complicated by physiologic factors such as age and bone marrow activation due to physical activity and infection.

\section{PET/MRI IN MM}

In recent years, simultaneous PET/MRI platforms have become available for clinical use. These hybrid systems can combine the molecular data of PET with the anatomic and functional data of MRI. The benefits of simultaneous acquisition are that 2 previously separate examinations can now be performed in a single imaging session, there is improved registration between modalities, and dynamic PET and DCE MRI can be done simultaneously. The drawbacks of hybridizing PET with MRI rest mainly on issues related to attenuation correction of the PET data. MR-based attenuation correction does not take into account cortical bone; however, vendors and researchers are actively investigating the potential impact of this factor on quantitative evaluation of osseous lesions while working toward improved technology. With regard to workflow challenges, it is essential to focus on patient tolerance and comfort when designing WB PET/MRI protocols (41). It is advisable that MRI sequences be minimized to what is essential to answer the clinical or research question. In the absence of WB PET/ MRI, WB PET imaging and MRI of the spine and pelvis are recommended. Additionally, any known or suspected areas of disease involvement may be targeted for imaging. PET/MRI protocols that are being optimized at our institution for prospective use in patients with MGUS, smoldering MM, and MM are summarized in Table 1. Figure 2 is an example of a fused PET/MR image showing an active site of MM involvement in a lumbar vertebral body. Studies evaluating PET/ MRI as a diagnostic tool for MM will provide more insight into the benefits of this promising imaging platform.

TABLE 1

MRI Sequences to Include in WB PET/MRI Evaluation of Marrow Lesions

\begin{tabular}{|c|c|}
\hline Sequence & Recommended use \\
\hline T1-weighted turbo spin echo & Evaluation of cortex (normally dark) and marrow infiltration (marrow darker than normal) \\
\hline $\begin{array}{l}\text { Contrast-enhanced T2-weighted fat } \\
\text { suppression }\end{array}$ & $\begin{array}{l}\text { Evaluation for marrow edema and replacement; a T2-weighted fat-suppressed sequence (areas of edema } \\
\text { and replacement are often brighter than background fat-containing marrow) }\end{array}$ \\
\hline $\begin{array}{l}\text { T2-weighted half-Fourier acquisition } \\
\text { single-shot fast spin echo }\end{array}$ & $\begin{array}{l}\text { Evaluation of the full body and anatomic detail on organs and soft tissues; a fast-acquisition T2-weighted } \\
\text { sequence }\end{array}$ \\
\hline ADC DWI* & Evaluation of lesion cellularity; a possible biomarker of treatment response \\
\hline DCE MRI* & $\begin{array}{l}\text { Evaluation of limited regions such as in the case of a plasmacytoma; a surrogate for perfusion and } \\
\text { permeability }\end{array}$ \\
\hline $\begin{array}{l}\text { Attenuation-corrected T1-weighted } \\
\text { Dixon }\end{array}$ & $\begin{array}{l}\text { Creation of a } \mu \text {-map for attenuation correction of PET data; a dual-echo gradient recalled echo sequence } \\
\text { that is acquired at in-phase and opposed-phase echo times with generation of fat-only and water-only } \\
\text { images }\end{array}$ \\
\hline \multicolumn{2}{|c|}{$\begin{array}{l}{ }^{*} \mathrm{ADC} \text { DWI and DCE MRI sequences may be applied in a more focused way to characterize specific sites of disease and potentially add value in assessin } \\
\text { tumor response. DCE is not a WB method. }\end{array}$} \\
\hline
\end{tabular}




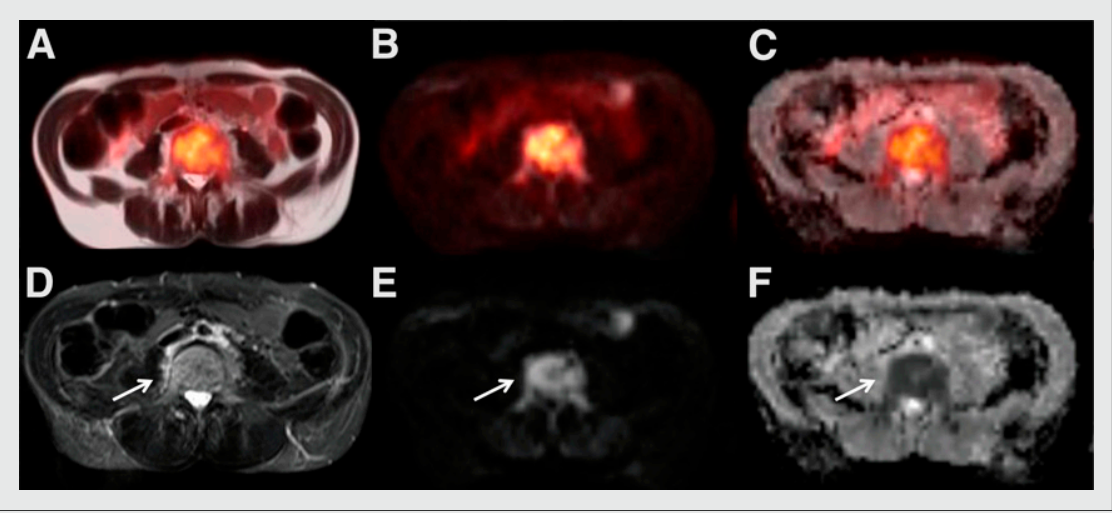

FIGURE 2. Axial MR and fused PET/MR images show active site of MM involvement in lumbar vertebral body (arrows): fused single-shot turbo spin echo T2-weighted MRI (A), fused DWI PET (B), fused ADC map PET (C), fat-suppressed turbo spin echo T2-weighted MRI (D), DWI $(E)$, and ADC (F). Bright signal intensity is seen on T2-weighted and diffusion images, with corresponding dark signal intensity on $A D C$ denoting restriction in diffusion-a correlate for increased cellular density. (Images obtained on Washington University clinical PET/MR scanner.)
14. Zamagni E, Patriarca F, Nanni C, et al. Prognostic relevance of 18-F FDG PET/CT in newly diagnosed multiple myeloma patients treated with up-front autologous transplantation. Blood. 2011;118:5989-5995.

15. Plathow C, Weber WA. Tumor cell metabolism imaging. J Nucl Med. 2008;49(suppl 2):43S-63S.

16. van Lammeren-Venema D, Regelink JC, Riphagen II, et al. ${ }^{18} \mathrm{~F}$-fluoro-deoxyglucose positron emission tomography in assessment of myeloma-related bone disease: a systematic review. Cancer. 2012;118:1971-1981.

17. Lyssiotis CA, Cantley LC. Acetate fuels the cancer engine. Cell. 2014;159:1492-1494.

18. Wang WQ, Zhao XY, Wang HY, et al. Increased fatty acid synthase as a potential therapeutic target in multiple myeloma. J Zhejiang Univ Sci B. 2008;9:441-447.

19. Okawa Y, Hideshima T, Ikeda H, et al. Fatty acid synthase is a novel therapeutic target in multiple myeloma. Br J Haematol. 2008;141:659-671.

20. Grassi I, Nanni C, Allegri V, et al. The clinical use of PET with ${ }^{11} \mathrm{C}$-acetate. Am J Nucl Med Mol Imaging. 2012;2:33-47.

21. Lin $\mathrm{C}, \mathrm{Ho} \mathrm{CL}, \mathrm{Ng} \mathrm{SH}$, et al. ${ }^{11} \mathrm{C}$-acetate as a new biomarker for PET/CT in patients with multiple myeloma: initial staging and postinduction response assessment. Eur J Nucl Med Mol Imaging. 2014;41: 41-49.

\section{CONCLUSION}

Imaging with PET and MRI using molecularly targeted probes is a promising precision medicine platform that might successfully address the clinical ambiguities of myeloma spectrum diseases.

\section{DISCLOSURE}

This work is supported by 1R01CA176221 and CTSA UL1 TR000448 to Monica Shokeen. No other potential conflict of interest relevant to this article was reported.

\section{ACKNOWLEDGMENTS}

We thank Drs. Walter J. Akers, Michael H. Tomasson, Jon McConathy, Farrokh Dehdashti, Katherine N. Weilbaecher, and Francesca Fontana for helpful discussions.

\section{REFERENCES}

1. Kuehl WM, Bergsagel PL. Molecular pathogenesis of multiple myeloma and its premalignant precursor. J Clin Invest. 2012;122:3456-3463.

2. Roodman GD. Pathogenesis of myeloma bone disease. J Cell Biochem. 2010;109:283-291.

3. Rajkumar SV, Dimopoulos MA, Palumbo A, et al. International Myeloma Working Group updated criteria for the diagnosis of multiple myeloma. Lancet Oncol. 2014;15:e538-e548.

4. Teitelbaum A, Ba-Mancini A, Huang H, Henk HJ. Health care costs and resource utilization, including patient burden, associated with novel-agent-based treatment versus other therapies for multiple myeloma: findings using real-world claims data. Oncologist. 2013;18:37-45.

5. Kristinsson SY, Anderson WF, Landgren O. Improved long-term survival in multiple myeloma up to the age of 80 years. Leukemia. 2014;28:1346-1348.

6. Anderson KC. Therapeutic advances in relapsed or refractory multiple myeloma. J Natl Compr Canc Netw. 2013;11:676-679.

7. Kyle RA, Gertz MA, Witzig TE, et al. Review of 1027 patients with newly diagnosed multiple myeloma. Mayo Clin Proc. 2003;78:21-33.

8. Wang TF, Ahluwalia R, Fiala MA, et al. The characteristics and outcomes of patients with multiple myeloma dual refractory or intolerant to bortezomib and lenalidomide in the era of carfilzomib and pomalidomide. Leuk Lymphoma. 2014;55:337-341.

9. Pianko MJ, Terpos E, Roodman GD, et al. Whole-body low-dose computed tomography and advanced imaging techniques for multiple myeloma bone disease. Clin Cancer Res. 2014;20:5888-5897.

10. Greipp PR, San Miguel J, Durie BG, et al. International staging system for multiple myeloma. J Clin Oncol. 2005;23:3412-3420.

11. Healy CF, Murray JG, Eustace SJ, et al. Multiple myeloma: a review of imaging features and radiological techniques. Bone Marrow Res. 2011;2011:583439.

12. Princewill K, Kyere S, Awan O, et al. Multiple myeloma lesion detection with whole body CT versus radiographic skeletal survey. Cancer Invest. 2013;31:206-211.

13. Kröpil P, Fenk R, Fritz LB, et al. Comparison of whole-body 64-slice multidetector computed tomography and conventional radiography in staging of multiple myeloma. Eur Radiol. 2008;18:51-58.
22. Ho CL, Chen S, Leung YL, et al. ${ }^{11} \mathrm{C}$-acetate PET/CT for metabolic characterization of multiple myeloma: a comparative study with ${ }^{18} \mathrm{~F}-\mathrm{FDG}$ PET/CT. J Nucl Med. 2014;55:749-752.

23. Nanni C, Zamagni E, Cavo M, et al. ${ }^{11} \mathrm{C}$-choline vs. ${ }^{18} \mathrm{~F}$-FDG PET/CT in assessing bone involvement in patients with multiple myeloma. World J Surg Oncol. 2007;5:68

24. Calabria F, Chiaravalloti A, Schillaci O. ${ }^{18} \mathrm{~F}$-choline PET/CT pitfalls in image interpretation: an update on 300 examined patients with prostate cancer. Clin Nucl Med. 2014:39:122-130.

25. Huang C, McConathy J. Fluorine-18 labeled amino acids for oncologic imaging with positron emission tomography. Curr Top Med Chem. 2013;13:871-891.

26. Nakamoto Y, Kurihara K, Nishizawa M, et al. Clinical value of ${ }^{11} \mathrm{C}$-methionine PET/CT in patients with plasma cell malignancy: comparison with ${ }^{18} \mathrm{~F}-\mathrm{FDG}$ PET/CT. Eur J Nucl Med Mol Imaging. 2013;40:708-715.

27. Luckerath K, Lapa C, Spahmann A, et al. Targeting paraprotein biosynthesis for noninvasive characterization of myeloma biology. PLoS One. 2013;8:e84840.

28. Isoda A, Kaira K, Iwashina M, et al. Expression of L-type amino acid transporter 1 (LAT1) as a prognostic and therapeutic indicator in multiple myeloma. Cancer Sci. 2014;105:1496-1502.

29. Shimaoka M, Springer TA. Therapeutic antagonists and conformational regulation of integrin function. Nat Rev Drug Discov. 2003;2:703-716.

30. Soodgupta D, Hurchla MA, Jiang M, et al. Very late antigen-4 ( $\alpha_{4} \beta_{1}$ integrin) targeted PET imaging of multiple myeloma. PLoS One. 2013;8:e55841.

31. Philipp-Abbrederis K, Herrmann K, Knop S, et al. In vivo molecular imaging of chemokine receptor CXCR4 expression in patients with advanced multiple myeloma. EMBO Mol Med. 2015;7:477-487.

32. Dutoit JC, Vanderkerken MA, Verstraete KL. Value of whole body MRI and dynamic contrast enhanced MRI in the diagnosis, follow-up and evaluation of disease activity and extent in multiple myeloma. Eur J Radiol. 2013;82:1444-1452.

33. Kristinsson SY, Minter AR, Korde N, Tan E, Landgren O. Bone disease in multiple myeloma and precursor disease: novel diagnostic approaches and implications on clinical management. Expert Rev Mol Diagn. 2011;11:593-603.

34. Hillengass J, Fechtner K, Weber MA, et al. Prognostic significance of focal lesions in whole-body magnetic resonance imaging in patients with asymptomatic multiple myeloma. J Clin Oncol. 2010;28:1606-1610.

35. Bhutani M, Turkbey B, Tan E, et al. Bone marrow angiogenesis in myeloma and its precursor disease: a prospective clinical trial. Leukemia. 2014;28:413-416.

36. Blackledge MD, Collins DJ, Tunariu N, et al. Assessment of treatment response by total tumor volume and global apparent diffusion coefficient using diffusion-weighted MRI in patients with metastatic bone disease: a feasibility study. PLoS One. 2014;9:e91779.

37. Giles SL, Messiou C, Collins DJ, et al. Whole-body diffusion-weighted MR imaging for assessment of treatment response in myeloma. Radiology. 2014;271:785-794.

38. Horger M, Weisel K, Horger W, et al. Whole-body diffusion-weighted MRI with apparent diffusion coefficient mapping for early response monitoring in multiple myeloma: preliminary results. AJR. 2011;196:W790-W795.

39. Hillengass J, Bauerle T, Bartl R, et al. Diffusion-weighted imaging for non-invasive and quantitative monitoring of bone marrow infiltration in patients with monoclonal plasma cell disease: a comparative study with histology. Br J Haematol. 2011;153:721-728.

40. Messiou C, Giles S, Collins DJ, et al. Assessing response of myeloma bone disease with diffusion-weighted MRI. Br J Radiol. 2012;85:e1198-e1203.

41. Martinez-Moller A, Eiber M, Nekolla SG, et al. Workflow and scan protocol considerations for integrated whole-body PET/MRI in oncology. $\mathrm{J} \mathrm{Nucl} \mathrm{Med.}$ 2012;53:1415-1426. 